\title{
THRESHING IN ACTION: THE TIDAL DISRUPTION OF A DWARF GALAXY BY THE HYDRA I CLUSTER*
}

\author{
Andreas Koch ${ }^{1}$, Andreas Burkert ${ }^{2,3,8}$, R. Michael Rich ${ }^{4}$, Michelle L. M. Collins ${ }^{5}$, Christine S. Black ${ }^{4}$, \\ Michael HilKer $^{6}$, AND ANDREW J. Benson ${ }^{7}$ \\ ${ }^{1}$ Zentrum für Astronomie der Universität Heidelberg, Landessternwarte, Königstuhl 12, D-69117 Heidelberg, Germany; akoch@1sw.uni-heidelberg.de \\ ${ }^{2}$ Universitätssternwarte der Ludwig-Maximilians Universität, Scheinerstr. 1, D-81679 München, Germany \\ ${ }^{3}$ Max-Planck Institute for Extraterrestrial Physics, Giessenbachstrasse, D-85748 Garching, Germany \\ ${ }^{4}$ Department of Physics and Astronomy, University of California Los Angeles, Los Angeles, CA, USA \\ ${ }^{5}$ Max-Planck-Institut für Astronomie, Königstuhl 17, D-69117 Heidelberg, Germany \\ ${ }^{6}$ European Southern Observatory, Karl-Schwarzschild-Strasse 2, D-85748 Garching, Germany \\ ${ }^{7}$ Department of Astronomy, Caltech, Pasadena, CA, USA \\ Received 2012 March 28; accepted 2012 July 11; published 2012 July 25
}

\begin{abstract}
We report on the discovery of strong tidal features around a dwarf spheroidal galaxy in the Hydra I galaxy cluster, indicating its ongoing tidal disruption. This very low surface brightness object, HCC-087, was originally classified as an early-type dwarf in the Hydra Cluster Catalogue (HCC), but our re-analysis of the ESO-VLT/FORS images of the HCC unearthed a clear indication of an S-shaped morphology and a large spatial extent. Its shape, luminosity $\left(M_{V}=-11.6 \mathrm{mag}\right.$ ), and physical size (at a half-light radius of $3.1 \mathrm{kpc}$ and a full length of $\sim 5.9 \mathrm{kpc}$ ) are comparable to the recently discovered NGC 4449B and the Sagittarius dwarf spheroidal, all of which are undergoing clear tidal disruption. Aided by $N$-body simulations we argue that HCC- 087 is currently at its first apocenter, at $150 \mathrm{kpc}$, around the cluster center and that it is being tidally disrupted by the galaxy cluster's potential itself. An interaction with the nearby (50 kpc) S0 cluster galaxy HCC-005, at $M_{*} \sim 3 \times 10^{10} M_{\odot}$ is rather unlikely, as this constellation requires a significant amount of dynamical friction and thus low relative velocities. The S-shaped morphology and large spatial extent of the satellite would, however, also appear if HCC-087 would orbit the cluster center. These features appear to be characteristic properties of satellites that are seen in the process of being tidally disrupted, independent of the environment of the destruction. An important finding of our simulations is an orientation of the tidal tails perpendicular to the orbit.
\end{abstract}

Key words: galaxies: clusters: individual (Hydra) - galaxies: dwarf - galaxies: individual (HCC-087, NGC 4449B) - galaxies: interactions - galaxies: structure

Online-only material: color figures

\section{INTRODUCTION}

Tidal sculpting and the disruption of satellite galaxies is a prevalent source for the hierarchical buildup of larger galaxies' halos (e.g., Searle \& Zinn 1978). The action of tides in the Local Group (LG) is in fact observed through the accretion of the Sagittarius galaxy (Ibata et al. 1994) and Andromeda's giant stellar stream (Ibata et al. 2001). Over the past years, tidal features manifested as low surface brightness structures surrounding nearby galaxies in the Local Volume (e.g., MartínezDelgado et al. 2010), and out to higher redshifts (Forbes et al. 2003; Sasaki et al. 2007; Arnaboldi et al. 2012), lending strong support to the hierarchical scenario of galaxy formation (e.g., Boylan-Kolchin et al. 2010).

The recent discovery of a disrupted satellite to the Magellanic irregular starburst galaxy NGC 4449 (Rich et al. 2012; MartínezDelgado et al. 2012) provides the first evidence that such accretion events already take place on remarkably small scales. This satellite exhibits an S-shaped morphology that is observed and predicted for tidally disrupting star clusters and galaxies (e.g., Capuzzo-Dolcetta et al. 2005; Peñarrubia et al. 2009). From a modeling point of view, tidal effects induce significant variations of a systems' characteristic radius (e.g., the half-light radius, $\left.r_{h}\right)$. For instance, it has been noted that the brighter

\footnotetext{
* Based on observations carried out at the European Southern Observatory under proposal number 65.N-0459(A).

8 Max-Planck Fellow.
}

satellite galaxies to M31 are more extended than their Milky Way (MW) counterparts-possibly induced by its massive disk (Hammer et al. 2007; Peñarrubia et al. 2010; cf. Richardson et al. 2011).

In this context, scaling relations for "dynamically hot" systems, i.e., those supported by random motions, provide an important tool for studying the underlying physics of structure formation (e.g., Gilmore et al. 2007; Dabringhausen et al. 2008; Misgeld \& Hilker 2011; Brasseur et al. 2011). Correlations between, say, luminosity and radius were found over a wide mass range, from giant ellipticals down to the dwarf galaxy regime. Moreover, star clusters and ultracompact dwarfs (UCDs), i.e., low-luminosity, spatially concentrated objects, are clearly differentiated in this parameter space. A characterization of extended, low surface brightness objects, however, is still incomplete, ${ }^{9}$ while those objects hold important clues to the environmental effects governing galaxy evolution.

Of further interest is the evolution of the galaxies themselves as they undergo threshing in the potential of their hosts: for instance, this way a transformation of a late-type spiral with a nuclear star cluster $(\mathrm{Sc}, \mathrm{N})$ into nucleated dwarfs can occur, until they may further morph into UCDs (e.g., Goerdt et al. 2008), which, in turn, may play an important role in the buildup of massive globular cluster systems. Clearly, this

\footnotetext{
9 It should be noted, however, that such scaling relations often hold a strong detection bias against extended, low surface brightness targets. For instance, the surface brightness detection limit of the Sloan Digital Sky Survey lies at $\mu_{V} \sim 30 \mathrm{mag} \operatorname{arcsec}^{-2}$.
} 


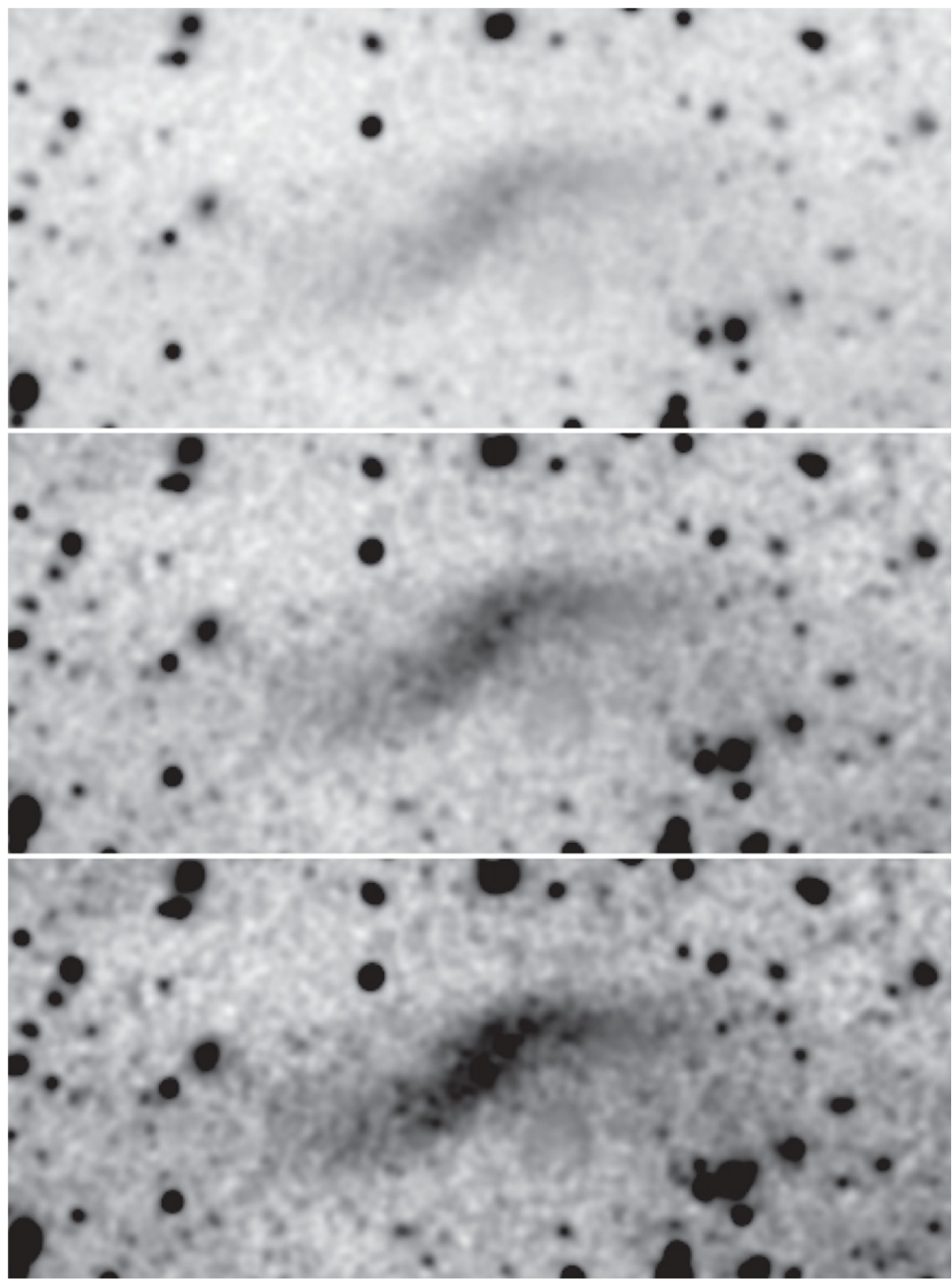

Figure 1. Co-added $V$ and $I$ images of HCC-087 using different stretches to enhance its morphology. These images cover $1.6 \times 00^{\prime} \cdot 7(\sim 22 \times 10 \mathrm{kpc})$ and were smoothed with a Gaussian kernel. Some bright stars close to the galaxy were point-spread-function-subtracted from the images.

complex interplay emphasizes the importance of studying tidally disrupting systems for a deeper understanding of structure formation. Unfortunately, the timescales for such interactions are yet poorly known and basically framed only by simulations (e.g., Peñarrubia et al. 2008, 2009).

Here we report on the discovery of an extended, earlytype galaxy in the Hydra I Cluster ( $\equiv$ A1060) that shows clear evidence of tidal disruption. Although its shape and luminosity parameters have been listed in the Hydra Cluster Catalogue (HCC; Misgeld et al. 2008, hereafter M08), its distorted appearance had so far eluded detection. No other such remarkable object has been found in this cluster, making it a unique object in the Local Volume, together with NGC 4449B. Hydra I is an evolved and dynamically relaxed, yet poor, galaxy cluster, dominated by early-type galaxies, at a distance of 47.2 Mpc (Misgeld et al. 2011).

\section{DATA}

The images presented herein were first published in Mieske et al. (2005) and M08. In brief, the exposures were taken with the Very Large Telescope (VLT)/FORS1 at ESO/Paranal in 2000 April. "Field 6," which also contains HCC-087, was observed in the $I$ band $(9 \times 330$ s) on 2000 April 5 and on 2000 April 6 in the $V$ band $(3 \times 480 \mathrm{~s})$. The conditions were mostly photometric, at an average seeing of 0 ' 6 in both $V$ and $I$ bands. For details on the photometric calibrations of those data we refer the reader to Mieske et al. (2005) and M08. Figures 1 and 2 show the relevant image of the field surrounding HCC-087.

M08 performed (elliptical) isophote fitting on locally skysubtracted images. Coupled with visual inspection, HCC-087 was originally classified as an early-type dwarf galaxy and its photometric and morphological parameters are $\mu_{0}=$ $26.3 \mathrm{mag} \mathrm{arcsec}^{-2}$ and $R_{\text {eff }}=13^{\prime \prime} .7$ (Table 1). At an estimated $M_{V}$ of $-11.6 \mathrm{mag}$, it is comparable to the luminous MW satellite Leo I (e.g., Koch 2009).

Its extraordinarily large radius of $3.1 \mathrm{kpc}$ (note that the average radius of the remainder of the HCC dwarfs lies at $650 \mathrm{pc}$ ) and its similarity with the strongly tidally affected NGC 4449B (Rich et al. 2012) prompted us to revisit those images, whereupon the tidal nature of HCC-087 became clear. We emphasize that the clearly distorted shape is visible on both the $V$ - and $I$-band images, ruling out an instrumental artifact. Unfortunately, no radial velocity measurement is available for 


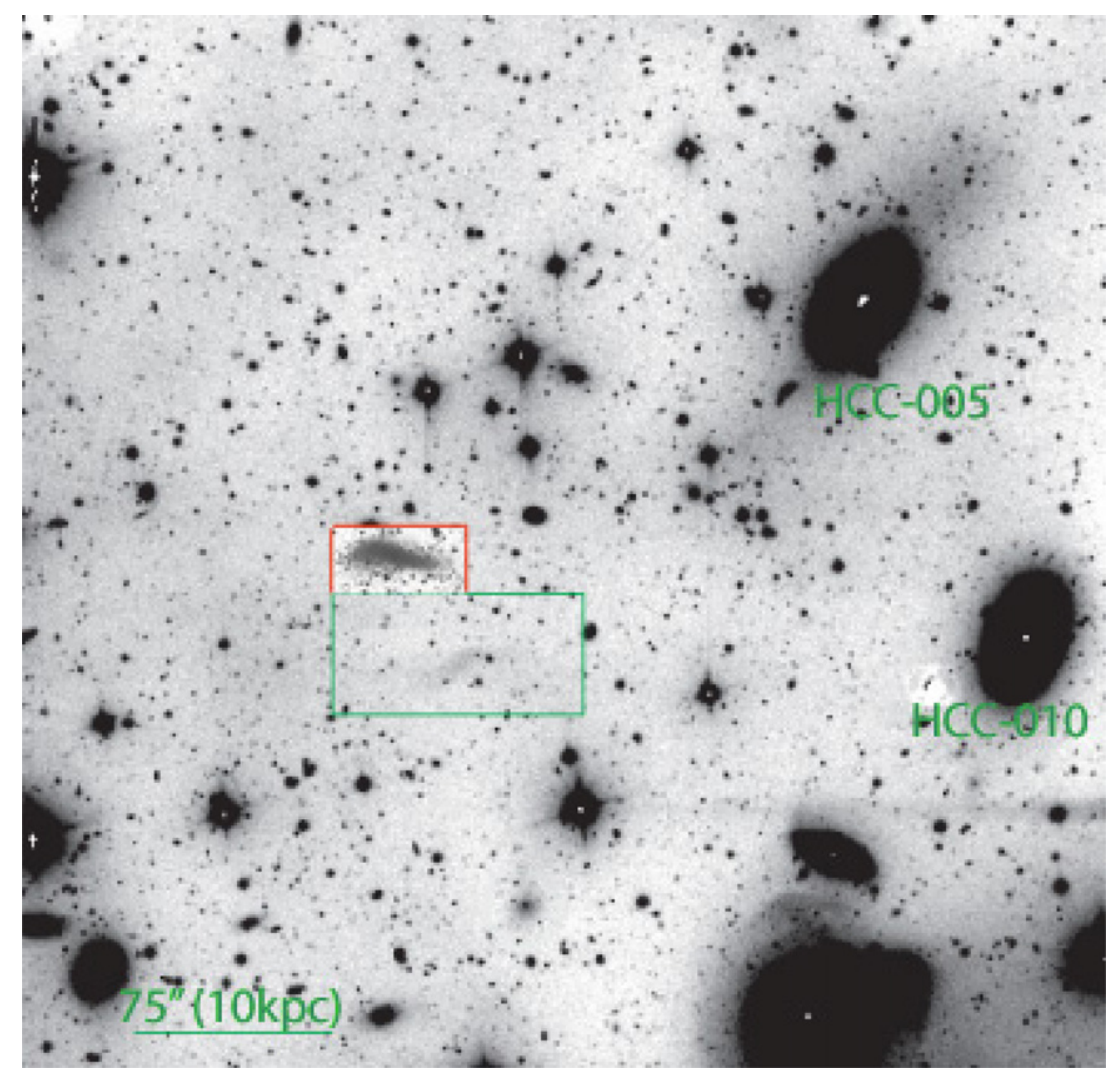

Figure 2. 6.8 $\times 6^{\prime} 8(\sim 93 \mathrm{kpc} \times 93 \mathrm{kpc}) V$-band image of the region around HCC-087 with the section from Figure 1 highlighted as a green box. The red-boxed inset shows NGC 4449B from Rich et al. (2012), scaled to the physical size at the distance of HCC-087. The scale bar indicates $75^{\prime \prime}$ (10 kpc at the distance of Hydra I). North is up and east is to the left. We also label HCC-005 and HCC-010, as discussed in the text. Not visible in this field are Hydra's main galaxies NGC 3309, 3311, and 3312 .

(A color version of this figure is available in the online journal.)

Table 1

Properties of HCC-087 and NGC 4449B

\begin{tabular}{lcc}
\hline \hline Parameter & HCC-087 & NGC 4449B \\
\hline$\alpha, \delta(\mathrm{J} 2000.0)$ & M08 & Rich et al. (2012) \\
$V, V-I(\mathrm{mag})$ & $10: 36: 39.0,-27: 21: 25.5$ & $12: 28: 45,43: 57: 44$ \\
$M_{V}(\mathrm{mag})$ & $-11.62 \pm 0.09$ & $14.88,0.48(\mathrm{~g}-\mathrm{r})$ \\
$\mu_{0}\left(\mathrm{mag} \mathrm{arcsec}^{-2}\right)$ & $26.28 \pm 0.05$ & $-13.03 \pm 0.10$ \\
$\Sigma_{0}\left(L_{\odot} \mathrm{pc}^{-2}\right)$ & $0.89 \pm 0.04$ & $25.5 \pm 0.05$ \\
$\mathrm{r}_{\mathrm{h}}(\operatorname{arcsec})$ & $13.65 \pm 4.62$ & $0.44 \pm 0.02$ \\
$\mathrm{r}_{\mathrm{h}}(\mathrm{kpc})^{\mathrm{a}}$ & $3.1 \pm 1.1$ & $147 \pm 14$ \\
\hline
\end{tabular}

Note. ${ }^{\text {a }}$ Adopting a distance modulus of 33.37 mag for HCC-087 (Misgeld et al. 2011) and of 27.91 for NGC 4449B (Annibali et al. 2008).

this galaxy, nor is that straightforward to obtain, given the object's faintness. We note, however, that there is no current evidence of any fore- or background structures within $\sim 50 \mathrm{Mpc}$ relative to Hydra (e.g., Richter 1989; M08).

\section{ENVIRONMENT AND TIDAL DISRUPTION}

\subsection{Morphology}

HCC-087 shows a clear S-shaped morphology, as reproduced in models of tidally disrupting dwarf satellites; the tails are aligned east to west.

The effective radius of HCC-087 from an exponential profile fit is $3.1 \pm 1.1 \mathrm{kpc}$ (M08). This is about the same value found for the dwarf galaxy NGC 4449B (Rich et al. 2012) that is currently undergoing strong tidal disruption on its first encounter with the luminous Magellanic galaxy NGC $4449\left(M_{B}=-18.2 \mathrm{mag}\right)$. Moreover, this value exceeds that of the (bound region of the) Sagittarius dwarf by at least $1 \mathrm{kpc}$ (Majewski et al. 2003; Walker et al. 2009), rendering HCC-087 and NGC 4449B the largest such measurements for dwarf galaxies (Figure 3). Furthermore, we estimate that the main body of HCC-087 is approximately $24 . .5 \times 8^{\prime \prime}$, or $5.6 \times 1.8 \mathrm{kpc}$, in extent.

In fact, both HCC-087 and NGC 4449B exceed the average radii of cluster galaxies in the Local Volume, as sampled by the Hydra I and Centaurus cluster members and the M31 dwarf spheroidal companions, by more than $2 \sigma$ (accounting for measurement errors and the scatter in the $r_{h}-M_{V}$ plot). This is emphasized by the solid and dashed lines in Figure 3, which show the running median and $2 \sigma$ limits of these galaxies.

While the significantly greater extent of a number of brighter M31 dwarfs compared to those of the MW has been noted by McConnachie \& Irwin (2006), we label in Figure 3 the extreme M31 structural outlier, And XIX (McConnachie et al. 2008). Although it has been suggested that tidal interactions with the disk of M31 are a possible cause for the larger extent (e.g., Collins et al. 2011), an assessment of the tidal nature of this satellite has to await measurements of its kinematics.

\subsection{HCC-087 within the Hydra Cluster}

Lacking a redshift measurement and in the light of the richness of the cluster field surrounding HCC-087 it is difficult to unambiguously identify an obvious parent galaxy that is host 


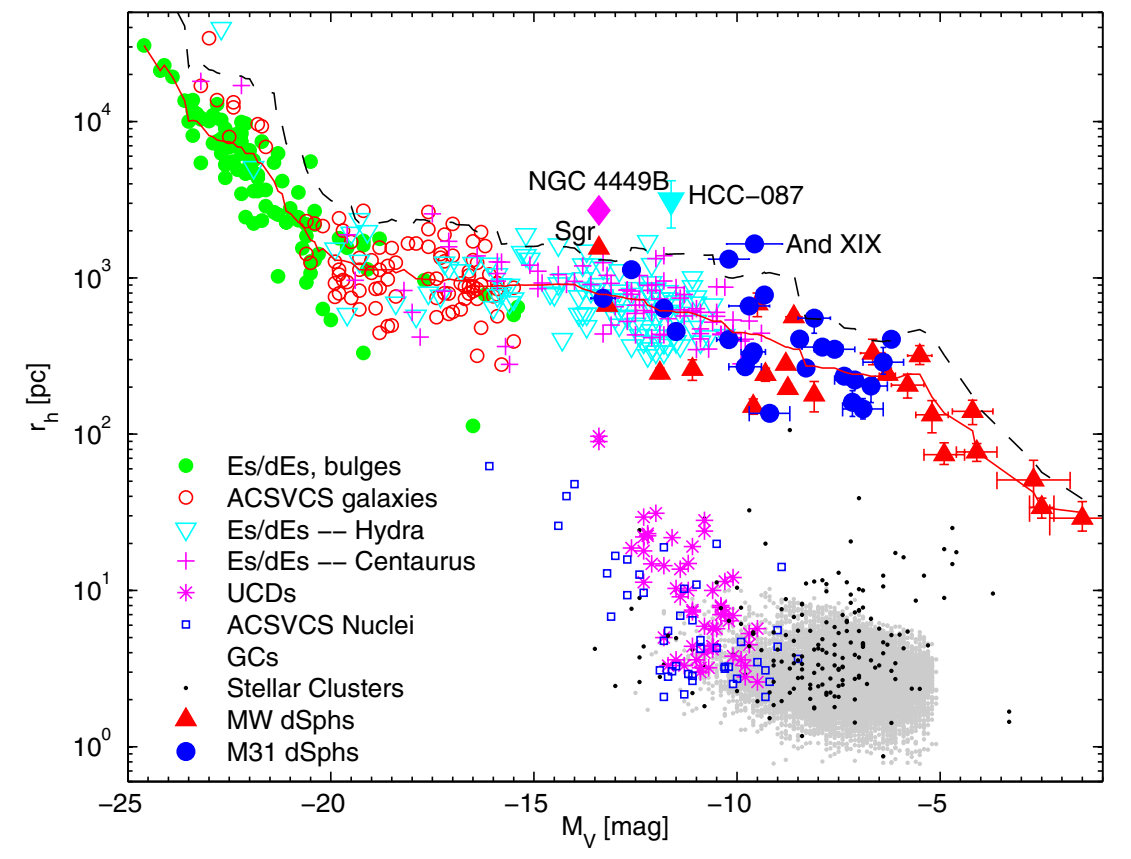

Figure 3. Radius-magnitude plot, adopting data and classifications from Misgeld \& Hilker (2011). Highlighted are the tidally disrupted HCC-087, NGC 4449B (Rich et al. 2012), and other remarkably extended galaxies. The solid line indicates the median relation defined by the upper branch in the $r_{h}-M_{V}$ plane and the dashed line delineates the $2 \sigma$ upper limit to this trend. Note that the decline at the faint end is purely a detection bias, reflecting the surface brightness limit.

(A color version of this figure is available in the online journal.)

to interactions with this dwarf galaxy. This is aggravated by the fact that the tails are seen in projection, while, arguing about the entire Hydra I cluster, we are in fact dealing with a threedimensional structure.

We note a clustering of several elliptical galaxies NW to SW of $\mathrm{HCC}-087$ and at projected distances of 35-50 kpc in Figure 2, but none of the Hydra censuses lists any member systems closer than 30 kpc (Richter 1989; Christlein \& Zabludoff 2003; HCC). It could be attractive to associate the brightest of the ellipticals in the SW corner of Figure 2 with HCC-087 as this object is surrounded by a pronounced shell, indicative of tidal interactions (e.g., Mori \& Rich 2008; McConnachie et al. 2009; Rich et al. 2012). However, this object lies at a redshift of $z=0.03$ (Smith et al. 2004) and, together with the galaxy $1^{\prime}$ to its north, is a confirmed non-member of the Hydra cluster and thus can be ruled out as interacting partner.

HCC-087 lies at a projected $140 \mathrm{kpc}$ from the cluster's primary galaxies, NGC 3309 (E3) and NGC 3311 (S0), and at $170 \mathrm{kpc}$ from the $S a b$ LINER galaxy NGC 3312 (not pictured in Figure 2). This is comparable to the location of the Hercules dwarf spheroidal satellite within the MW halo (although the LG clearly provides a different environment), which has been suggested to be a stellar, tidal stream in the process of formation (Martin \& Jin 2010). This provides the same order of magnitude as is seen in interacting dwarf-giant galaxies at higher redshifts (Sasaki et al. 2007).

This is in contrast to NGC 4449B lying at $9 \mathrm{kpc}$ from NGC 4449 (albeit lower mass than the luminous Hydra galaxies) and being on its first infall path toward its disruptive host. Rich et al. (2012) suggested from the appearance of this dwarf's tidal tails that it must be on a highly eccentric orbit and is currently 5-10 crossing times (or $200 \mathrm{Myr}$ ) past perigalacticon; this is also the timescale needed for transversing the (projected) distance between the partner and the dwarf galaxy under reasonable assumptions for its orbital motion.
There are two other HCC early-type galaxies, $\sim 50 \mathrm{kpc}$ in projection to the $\mathrm{W}$ and $\mathrm{NW}$ of the dwarf-HCC-010 and HCC-005, respectively, with stellar masses of a few times $10^{10} M_{\odot}$ (Misgeld \& Hilker 2011). Based on the orientation of the tidal tails and aided by our $N$-body simulations (Section 3.3), we will continue by considering both the cluster's central potential and $\mathrm{HCC}-005$ as possible hosts to the tidal disruption of HCC-087, as we elaborate in the following.

\subsection{Simulations of Tidal Disruption}

A clearly boxy shape of the envelope (third panel of Figure 1) is reminiscent of simulations of tidally disrupting galaxies (e.g., Bekki et al. 2001; Peñarrubia et al. 2008), all of which bear closest resemblance with HCC-087 near pericenter after an interaction timescale of the order of $500 \mathrm{Myr}$. The lack of an obvious, very nearby parent for HCC-087, however, would be worrisome if it was at pericenter, even more so considering the ephemerality of the tidal arm features in those simulations: the S-shaped morphology of threshed galaxies in the simulations of, say, Peñarrubia et al. (2008) become unstable on a timescale of a few $10^{8}$ yr.

Thus we set up a new suite of simulations (A. Burkert et al., in preparation), in which we can reproduce the appearance of HCC-087 in an environment as the Hydra cluster.

\subsubsection{HCC-087 orbiting HCC-005}

In practice, we model the dwarf as a one-component ${ }^{10}$ Hernquist (1990) profile with $r=0.5 \mathrm{kpc}$ and $1 \times 10^{7} M_{\odot}$

\footnotetext{
10 Simulations with a concentrated stellar component embedded in an extended dark matter halo reach identical conclusions, i.e., the stars trace the disruption of the dark halo and outline the observed, characteristic S-shape. A prerequisite is an a priori large extent of the stellar component.
} 

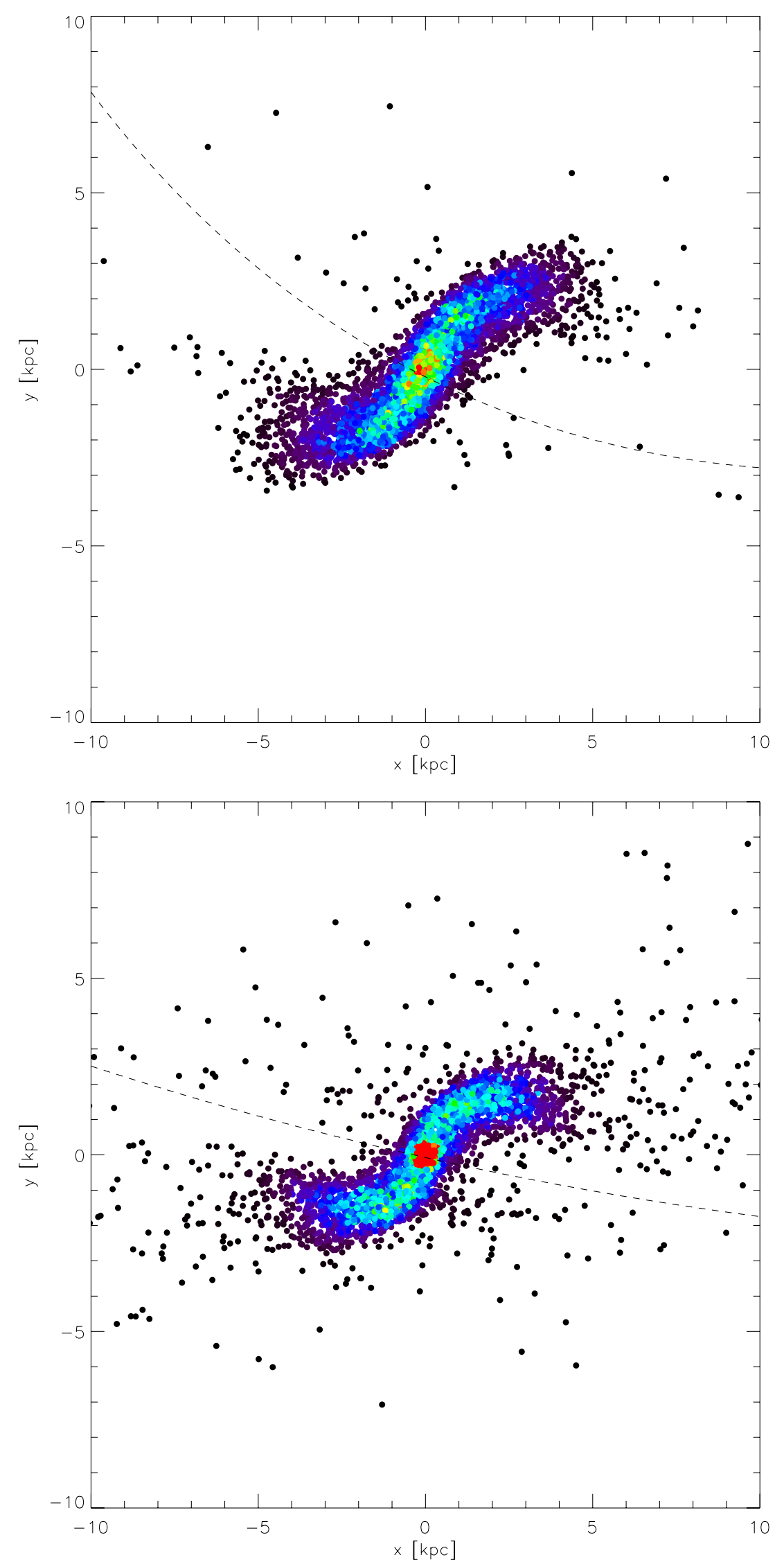

Figure 4. Snapshot of the simulation of HCC-087 orbiting the Hydra I galaxy HCC-005 (top panel) and the Hydra cluster center (bottom panel), color-coded by stellar density. The observed morphology is reproduced at first apocenter, each after $\sim 0.8 \mathrm{Gyr}$.

(A color version of this figure is available in the online journal.) 
(Misgeld \& Hilker 2011) ${ }^{11}$ and place it in an orbit around a massive, HCC-005-like galaxy with $M_{\text {tot }}=5 \times 10^{11} M_{\odot}$ (Misgeld \& Hilker 2011). ${ }^{12}$

Surprisingly, our simulations indicate that an S-shape of the disrupting satellite, just as observed for HCC-087, naturally emerges at the first apocenter (Figure 4), which, in the simulations, occurs at $50 \mathrm{kpc}$ - coinciding with the (projected) presentday distance between HCC-087 and HCC-005. More strikingly, the orientation of the tidal tails at apocenter is perpendicular to the orbit.

Its having past the first apocenter passage at $50 \mathrm{kpc}$ then begs the question of the origin of HCC-087. We note that "first-infall" events are not uncommon in the LG as several high-velocity dwarf galaxies make their passages through the M31 or MW halos (Chapman et al. 2007; Majewski et al. 2007; Lépine et al. 2011). None of these, however, displays any evidence of tidal tails. Therefore, we investigated the possibility that the observed apocenter could be a result of dynamical friction, adopting a radius and mass-to-light ratio as for $\mathrm{MW}$ dSphs of comparable luminosities (Koch et al. 2007). We find that dynamical friction is able to bring HCC-087 on a bound orbit as observed after one orbital period, while starting at the virial radius of the host galaxy. This requires, however, a very small relative velocity of the interactors $\left(\sim 100 \mathrm{~km} \mathrm{~s}^{-1}\right)$, which is in contrast to the high virial velocity of the relaxed cluster ( $750 \mathrm{~km} \mathrm{~s}^{-1}$; M08).

\subsubsection{HCC-087 Orbiting the Hydra I Potential}

As an alternative, we placed HCC-087 on an eccentric and a circular orbit around the cluster center itself, represented by the location of the primary galaxy NGC 3311 (e.g., Arnaboldi et al. 2012). To this end, we computed the total baryonic mass of Hydra I within $150 \mathrm{kpc}$ (the present position of HCC-087), using the data of Christlein \& Zabludoff, as $2 \times 10^{12} M_{\odot}$ and a dark matter mass 10-25 times as large (we note that the results turn out insensitive to the exact value). This setup reproduces Hydra's virial velocity at $750 \mathrm{~km} \mathrm{~s}^{-1}$ and places HCC-087 at a starting distance of $150 \mathrm{kpc}$ with a pericenter of $50 \mathrm{kpc}$ in the eccentric case (A. Burkert et al., in preparation).

As a result, this scenario is also able to reproduce the morphology, orientation, and spatial extent of HCC-087, in full accord with Figure 4. However, contrary to the interaction with HCC-005 (Section 3.3.1), a disruption by the cluster itself is bolstered by the higher relative velocity of the $\mathrm{dSph}$ and the parent, which is more realistic given the high velocity dispersion of the cluster. Nonetheless, the comparison with the setup in Section 3.3.1 and the similar case of NGC 4449B indicates that the morphology of the object is largely independent of the host of the disruption. Likewise, the shape and density profile of the satellite at first apocenter is close to indistinguishable between the cases of an orbit around HCC-005 and the cluster center (Figure 4).

\section{SUMMARY}

We report on the re-analysis of deep imaging data of the Hydra I galaxy cluster, on which we detect the clear signatures of tidal disruption on one of the HCC early-type dwarfs. Its large

\footnotetext{
11 We also ran simulations with Plummer profiles and verified that the results do not depend on the shape of the profile as long as the satellite is strongly tidally perturbed during pericenter passage.

12 Note that this is the total mass, accounting for the galaxy's dark halo. This value is consistent with the observed stellar mass of $3.4 \times 10^{10} M_{\odot}$ (Misgeld $\&$ Hilker 2011), assuming a dark-matter-to-baryon fraction similar to the MW.
}

spatial extent of $3.1 \mathrm{kpc}$ renders it one of the most extended dwarf galaxies in the Local Volume, in line with NGC 4449B, And XIX, and our in-house, prime example, Sagittarius.

HCC-005 lies roughly on a line connecting HCC-087 and the cluster center-a constellation that will inevitably put HCC-087's morphological evolution into a predicament. Overall, we emphasize the significance of its disruption in that the cluster center exerts such a devastating effect on the satellite despite its large distance.

Aided by a new suite of $N$-body simulations we argue that the dwarf's morphology is caused by orbiting the galaxy cluster center. An orientation of the tails perpendicular to the orbit is naturally reproduced at the current apocenter at $150 \mathrm{kpc}$ after an interaction timescale of $\sim 0.6 \mathrm{Gyr}$ on an eccentric orbit, and similarly for several Gyr when we assume an almost circular orbit.

While, morphologically, we cannot rule out that HCC-087 was disrupted by the closer $(50 \mathrm{kpc})$ early-type galaxy HCC-005, the very low relative velocity (less than $15 \%$ the cluster's virial velocity) required for such an interaction renders this scenario unlikely. Nonetheless, our simulations have quantitatively shown that the structure of the satellite alone is no unique indicator of the system it is orbiting.

A.K. thanks the Deutsche Forschungsgemeinschaft for funding from Emmy-Noether grant Ko 4161/1.

\section{REFERENCES}

Annibali, F., Aloisi, A., Mack, J., et al. 2008, AJ, 135, 1900

Arnaboldi, M., Ventimiglia, G., Iodice, E., Gerhard, O., \& Coccato, L. 2012, A\&A, in press (arXiv:1205.5289)

Bekki, K., Couch, W. J., \& Drinkwater, M. J. 2001, ApJ, 552, L105

Boylan-Kolchin, M., Springel, V., White, S. D. M., \& Jenkins, A. 2010, MNRAS, 406, 896

Brasseur, C. M., Martin, N. F., Macciò, A. V., Rix, H.-W., \& Kang, X. 2011, ApJ, 743, 179

Capuzzo-Dolcetta, R., Di Matteo, P., \& Miocchi, P. 2005, AJ, 129, 1906

Chapman, S. C., Peñarrubia, J., Ibata, R., et al. 2007, ApJ, 662, L79

Christlein, D., \& Zabludoff, A. 2003, Ap\&SS, 285, 197

Collins, M. L. M., Chapman, S. C., Rich, R. M., et al. 2011, MNRAS, 417, 1170

Dabringhausen, J., Hilker, M., \& Kroupa, P. 2008, MNRAS, 386, 864

Forbes, D. A., Beasley, M. A., Bekki, K., Brodie, J. P., \& Strader, J. 2003, Science, 301, 1217

Gilmore, G., Wilkinson, M. I., Wyse, R. F. G., et al. 2007, ApJ, 663, 948

Goerdt, T., Moore, B., Kazantzidis, S., et al. 2008, MNRAS, 385, 2136

Hammer, F., Puech, M., Chemin, L., Flores, H., \& Lehnert, M. D. 2007, ApJ, 662,322

Hernquist, L. 1990, ApJ, 356, 359

Ibata, R., Irwin, M., Lewis, G., Ferguson, A. M. N., \& Tanvir, N. 2001, Nature, 412, 49

Ibata, R. A., Gilmore, G., \& Irwin, M. J. 1994, Nature, 370, 194

Koch, A. 2009, Astron. Nachr., 330, 675

Koch, A., Wilkinson, M. I., Kleyna, J. T., et al. 2007, ApJ, 657, 241

Lépine, S., Koch, A., Rich, R. M., \& Kuijken, K. 2011, ApJ, 741, 100

Majewski, S. R., Beaton, R. L., Patterson, R. J., et al. 2007, ApJ, 670, L9

Majewski, S. R., Skrutskie, M. F., Weinberg, M. D., \& Ostheimer, J. C. 2003, ApJ, 599, 1082

Martin, N. F., \& Jin, S. 2010, ApJ, 721, 1333

Martínez-Delgado, D., Gabany, R. J., Crawford, K., et al. 2010, AJ, 140, 962

Martínez-Delgado, D., Romanowsky, A. J., Gabany, R. J., et al. 2012, ApJ, 748, L24

McConnachie, A. W., Huxor, A., Martin, N. F., et al. 2008, ApJ, 688, 1009

McConnachie, A. W., \& Irwin, M. J. 2006, MNRAS, 365, 1263

McConnachie, A. W., Irwin, M. J., Ibata, R. A., et al. 2009, Nature, 461, 66

Mieske, S., Hilker, M., \& Infante, L. 2005, A\&A, 438, 103

Misgeld, I., \& Hilker, M. 2011, MNRAS, 414, 3699

Misgeld, I., Mieske, S., \& Hilker, M. 2008, A\&A, 486, 697 (M08)

Misgeld, I., Mieske, S., Hilker, M., et al. 2011, A\&A, 531, A4 
Mori, M., \& Rich, R. M. 2008, ApJ, 674, L77

Peñarrubia, J., Benson, A. J., Walker, M. G., et al. 2010, MNRAS, 406, 1290

Peñarrubia, J., McConnachie, A. W., \& Navarro, J. F. 2008, ApJ, 672, 904

Peñarrubia, J., Navarro, J. F., McConnachie, A. W., \& Martin, N. F. 2009, ApJ, 698, 222

Rich, R. M., Collins, M. L. M., Black, C. M., et al. 2012, Nature, 482, 192
Richardson, J. C., Irwin, M. J., McConnachie, A. W., et al. 2011, ApJ, 732, 76 Richter, O.-G. 1989, A\&AS, 77, 237

Sasaki, S. S., Taniguchi, Y., Scoville, N., et al. 2007, ApJS, 172, 511

Searle, L., \& Zinn, R. 1978, ApJ, 225, 357

Smith, R. J., Hudson, M. J., Nelan, J. E., et al. 2004, AJ, 128, 1558

Walker, M. G., Mateo, M., Olszewski, E. W., et al. 2009, ApJ, 704, 1274 